\title{
PERIOD REGION DISTRIBUTIONS OF MCMULLEN FAMILY M SETS
}

\author{
ZHIXING LU, YUANYUAN SUN* and XIAOPENG HU \\ College of Computer Science and Technology \\ Dalian University of Technology, Dalian, P. R. China \\ *syuan@dlut.edu.cn
}

Received January 13, 2017

Revised April 14, 2017

Accepted April 20, 2017

Published June 6, 2017

\begin{abstract}
In this study, the period- $n$ steady regions of McMullen family M sets are discussed. The calculation methods of the numbers of period- $n$ steady regions are presented and the center points and boundaries of period- 1 are studied by complex dynamical system theory. In addition, the problem about the free critical points is discussed. The experimental results show that the free critical points do not influence the distributions of period- $n$ steady regions when $m=d$, which is proved theoretically.
\end{abstract}

Keywords: McMullen M Sets; Algebraic Analysis Method; Period- $n$; Steady Region.

\section{INTRODUCTION}

In 1975, Mandelbrot published his epochal work "The Fractal Geometry of Nature" 1 From then on, more and more studies have been carried out in the field of fractal theory $\sqrt[2-7]{7}$ Although the mathematics theory of fractal is not complete yet, it gives us fresh and creative thoughts of nature world and human society.

In recent years, there have been tremendous interests in the investigations of McMullen family.

\footnotetext{
${ }^{*}$ Corresponding author.
}

This is an Open Access article published by World Scientific Publishing Company. It is distributed under the terms of the Creative Commons Attribution 4.0 (CC-BY) License. Further distribution of this work is permitted, provided the original work is properly cited. 
Liu et al. 8 11 Blanchard et al. 12 17 Roesch 18 researched them widely and deeply, finding their plentiful and beautiful topology structures. The topology structures may contain Cantor set, Cantor ring, Sierpinski curve and so on.

In this paper,we discuss the period- $n$ steady regions of McMullen family $\mathrm{M}$ sets. We use complex dynamical system theory to analyze the period1 steady center points and boundaries and utilize the algebraic analysis method to calculate the number of period- $n(n \geq 2)$ steady regions. Then, we explore the structure symmetries of McMullen family $\mathrm{M}$ sets. At last, we present a drawing algorithm of $\mathrm{M}$ set to verify the conclusions and study the impact of different free critical points on steady regions.

\section{PERIOD STEADY REGION DISTRIBUTIONS FOR MCMULLEN FAMILY}

For McMullen family

$$
\begin{aligned}
& \cdots Z_{n+1}=Z_{n}^{m}+\frac{C}{Z^{d}}, \\
& C \in \mathbf{C}^{*}=\mathbf{C} \backslash\{0\}, \\
& \frac{1}{m}+\frac{1}{d}<1,
\end{aligned}
$$

we change it to

$$
\begin{aligned}
\cdots Z_{n+1} & =\frac{1}{Z_{n}^{d}}\left(Z_{n}^{m+d}+C\right), \\
C \in \mathbf{C}^{*} & =\mathbf{C} \backslash\{0\}, \\
\frac{1}{m}+\frac{1}{d} & <1,
\end{aligned}
$$

where $C=r e^{i \theta}, \theta \in[0,2 \pi)$. Our work is about the steady period property of McMullen family M sets. Firstly, we discuss the period-1 steady region.

Consider the one-dimensional complex map:

$$
Z_{n+1}=f\left(Z_{n}, C\right) .
$$

The steady condition of its period- $n$ orbit $\frac{19}{19}$ is

$$
\left|g_{N}\left(Z_{i}, C\right)\right| \leq 1,
$$

where

$$
g_{N}\left(Z_{i}, C\right)=\prod_{i=1}^{N} f_{Z}^{\prime}\left(Z_{i}, C\right) .
$$

Let $Z_{*}$ a point in period-1 region, then

$$
Z_{*}=\frac{1}{Z_{*}^{d}}\left(Z_{*}^{m+d}+C\right)
$$

and

$$
\begin{aligned}
g_{1}\left(Z_{*}, C\right) & =m Z_{*}^{m-1}-\frac{d C}{Z_{*}^{d+1}} \\
& =\frac{1}{Z_{*}^{d+1}}\left(m Z_{*}^{m+d}-d C\right) .
\end{aligned}
$$

Then we can carry out Theorem [1.

Theorem 1. The steady center points of period-1 regions of McMullen family $M$ sets are

$$
C=m d^{\frac{d+1}{m-1}}(d+m)^{\frac{m+d}{1-m}} e^{i \frac{d+1}{m-1} 2 k \pi}, \quad k \in Z .
$$

Proof. It follows form (7), let $g_{1}(Z, C)=0$, then $\frac{1}{Z_{*}^{d+1}}\left(m Z_{*}^{m+d}-d C\right)=0$. The result is $Z_{*}=$ $\left(\frac{d C}{m}\right)^{\frac{1}{m+d}}$ and $Z_{*} \neq 0$. If we plug $Z_{*}=\left(\frac{d C}{m}\right)^{\frac{1}{m+d}}$ back into $Z_{*}=Z_{*}^{m}+\frac{C}{Z_{*}^{d}}$, then $C$ is the period-1 steady center point. The detailed analysis is as follows:

$$
\begin{gathered}
Z_{*}^{d+1}=Z_{*}^{m+d}+C, \\
\left(\frac{d C}{m}\right)^{\frac{d+1}{m+d}}=\frac{d C}{m}+C, \\
\left(\frac{d}{m}\right)^{\frac{d+1}{m+d}} C^{\frac{d+1}{m+d}}=C\left(\frac{d}{m}+1\right),
\end{gathered}
$$

$$
\left(\frac{d}{m}\right)^{\frac{d+1}{m+d}}\left(\frac{m}{d+m}\right)=C^{\frac{m-1}{m+d}} .
$$

It is easy to show that

$$
C=m d^{\frac{d+1}{m-1}}(d+m)^{\frac{m+d}{1-m}} e^{i \frac{d+1}{m-1} 2 k \pi}, \quad k \in Z .
$$

The proof is completed.

It is apparent from (8) that the number of steady center points of period- 1 is determined by $m$ and $d$. In the proof above, if we let $g_{1}(Z, C)=0$ into $g(Z, C)=e^{i \theta}, \theta \in[0,2 \pi)$, we can obtain Theorem 2 .

Theorem 2. The boundaries of period-1 steady domain are

$$
C=\frac{m-e^{i \theta}}{m+d}\left[\frac{d+e^{i \theta}}{m+d}\right]^{\frac{d+1}{m-1}}, \quad \theta \in[0,2 \pi) .
$$

Proof. We evaluate the equations

$$
\left\{\begin{array}{l}
\frac{1}{Z_{*}^{d+1}}\left(m Z_{*}^{m+d}-d C\right)=e^{i \theta}, \\
Z_{*}=Z_{*}^{m}+\frac{C}{Z_{*}^{d}} .
\end{array}\right.
$$


Change $Z_{*}=Z_{*}^{m}+\frac{C}{Z_{*}^{d}}$ into $Z_{*}^{d+1}=Z_{*}^{m+d}+C$ and change $\frac{1}{Z_{*}^{d+1}}\left(m Z_{*}^{m+d}-d C\right)=e^{i \theta}$ into $m Z_{*}^{m+d}-$ $d C=e^{i \theta} Z_{*}^{d+1}$, then Eq. (12) can be converted into

$$
\left\{\begin{array}{l}
m Z_{*}^{m+d}-d C=e^{i \theta} Z_{*}^{d+1}, \\
Z_{*}^{d+1}=Z_{*}^{m+d}+C .
\end{array}\right.
$$

It is easy to see that

$$
C^{\frac{1-m}{d+1}}=\left[\frac{m+d}{m-e^{i \theta}}\right]^{\frac{m+d}{d+1}} \cdot \frac{m-e^{i \theta}}{d+e^{i \theta}}
$$

then

$$
\begin{aligned}
C & =\left[\frac{m+d}{m-e^{i \theta}}\right]^{\frac{m+d}{1-m}} \cdot\left[\frac{m-e^{i} \theta}{d+e^{i} \theta}\right]^{\frac{d+1}{1-m}} \\
& =\left[\frac{m+d}{m-e^{i \theta}}\right]^{\frac{m-1}{1-m}}\left[\frac{m+d}{m-e^{i \theta}}\right]^{\frac{d+1}{1-m}}\left[\frac{m-e^{i} \theta}{d+e^{i} \theta}\right]^{\frac{d+1}{1-m}} \\
& =\frac{m-e^{i \theta}}{m+d}\left[\frac{d+e^{i \theta}}{m+d}\right]^{\frac{d+1}{m-1}} .
\end{aligned}
$$

The proof is completed.

The steady center points and boundaries of period- 1 are easy to calculate. For the period- $n$ $(n \geq 2)$, we will use the algebraic analysis method to analyze the relation between period points and the roots of equation. Huang proposed the algebraic analysis method to solve the problem about the integer power polynomial map.20 Inspired by this, we exploit the method to analyze McMullen family which belongs to the rational power polynomial map.

For form (2), if there is an orbit of period $N$, then it is easy to obtain that

$$
\begin{gathered}
Z_{2}=\frac{1}{Z_{1}^{d}}\left(Z_{1}^{m+d}+\lambda\right), \quad Z_{3}=\frac{1}{Z_{2}^{d}}\left(Z_{2}^{m+d}+\lambda\right) \\
\ldots \\
Z_{N}=\frac{1}{Z_{N-1}^{d}}\left(Z_{N-1}^{m+d}+\lambda\right), \quad Z_{1}=\frac{1}{Z_{N}^{d}}\left(Z_{N}^{m+d}+\lambda\right) .
\end{gathered}
$$

The points of the orbit of period $N$ can be considered as the roots of $N$ times algebraic equation

$$
Z^{N}+a_{1} Z^{N-1}+\cdots+a_{N-1} Z+a_{N}=0 .
$$

Then how to calculate the points of period $N$ can be changed into the problem about the roots of an $N$ times algebraic equation.
Theorem 3. For the complex map (2), there exist orbits with period $N$, where $N$ is an arbitrary natural number $N=N_{1}^{k_{1}} N_{2}^{k_{2}} \cdots N_{r}^{k_{r}},\left(N_{1}<N_{2}<\cdots<\right.$ $N_{r}$ are prime numbers and $k_{1}, k_{2}, \ldots, k_{r}$ are whole numbers which are greater than one, $r$ is the number of different prime numbers). Then the number of period- $N$ orbits is

$$
\begin{aligned}
H_{N}= & \frac{1}{N}\left[(m+d)^{N}-\sum_{i=1}^{r}(m+d)^{e_{i}}\right. \\
& +\sum_{j=2}^{r} \sum_{i=1}^{j-1}(m+d)^{e_{i j}} \\
& \left.+\cdots+(-1)^{r}(m+d)^{e_{12} \cdots r}\right]
\end{aligned}
$$

where $e_{i}=\frac{N}{N_{i}}, e_{i j}=\frac{N}{N_{i} N_{j}}, \ldots e_{12 \cdots r}=N / \prod_{i=1}^{r} N_{i}$.

Proof. We proceed to prove this theorem. Let $\left\{Z_{i}\right\}_{i=1}^{N}$ be a period- $N$ orbit, then $\left\{Z_{i}\right\}_{i=1}^{N}$ should be satisfied with iterative equations of (16). Substitute $Z_{2}$ into $Z_{3}$, then

$$
\begin{aligned}
Z_{3}= & \frac{1}{\left(\frac{1}{Z_{1}^{d}}\left(Z_{1}^{m+d}+C\right)\right)^{d}} \\
& \times\left(\left(\frac{1}{Z_{1}^{d}}\left(Z_{1}^{m+d}+C\right)\right)^{m+d}+C\right) \\
= & \frac{Z_{1}^{d^{2}}}{\left(Z_{1}^{m+d}+C\right)^{d}} \\
& \times\left(\frac{1}{Z_{1}^{d(m+d)}}\left(Z_{1}^{m+d}+C\right)^{m+d}+C\right) .
\end{aligned}
$$

The highest degree of Eq. (19) is $Z_{1}^{(m+d)^{2}}$. Performing the same method, we substitute $Z_{3}$ into $Z_{4}$ and $Z_{4}$ into $Z_{5}$. We will have an algebraic equation whose highest degree is $(m+d)^{N}$. This algebraic equation has $(m+d)^{N}$ roots. The $(m+d)^{N}$ roots should contain the lower period orbits of all different factors of $N$. So, we have the equation below:

$$
\begin{aligned}
(m+d)^{N}= & N H_{N}+\sum_{i=1}^{r} e_{i} H_{e i}+\sum_{j=1}^{r} \sum_{i=2}^{i-1} e_{i j} H_{e i j} \\
& +\sum_{k=3}^{r} \sum_{j=2}^{k-1} \sum_{i=1}^{j-1} e_{i j k} H_{e i j k}+\cdots+
\end{aligned}
$$




$$
\begin{aligned}
= & \sum_{l_{1}=0}^{k_{1}} \sum_{l_{2}=0}^{k_{2}} \cdots \sum_{l_{r}=0}^{k_{r}} \\
& \times e_{N}\left(l_{1}, l_{2}, \ldots, l_{r}\right) H_{e_{N\left(l_{1}, l_{2}, \ldots, l_{r}\right)}}
\end{aligned}
$$

where $e_{N}\left(l_{1}, l_{2}, \ldots, l_{r}\right)=N /\left(N_{1}^{l_{1}} N_{2}^{l_{2}} \cdots N_{r}^{l_{r}}\right)$. Similarly,

$$
\begin{aligned}
(m+d)^{e_{i}}= & e_{i} H_{e_{i}}+\sum_{\substack{j=1 \\
j \neq i}}^{r} e_{i j} H_{e_{i j}} \\
& +\sum_{\substack{k=2 \\
j \neq i \neq k}}^{r} \sum_{j=1}^{k-1} e_{i j k} H_{e_{i j k}} \\
& +\cdots=\sum_{l_{0}=0}^{k_{1}} \ldots \sum_{l_{i}=0}^{k_{i-1}} \ldots \sum_{l_{r}=0}^{k_{r}} \\
& \times e_{e_{i}}\left(l_{1}, l_{2}, \ldots, l_{r}\right) H_{e_{e i}\left(l_{1}, l_{2}, \ldots, l_{r}\right)}, \\
(m+d)^{e_{i j}}= & e_{i j} H_{e_{i j}}+\sum_{\substack{k=1 \\
j \neq k \neq i}}^{r} e_{i j k} H_{e_{i j k}}+\cdots \\
= & \sum_{l_{1}=0}^{k_{1}} \ldots \sum_{l_{i}=0}^{k_{i=1} \ldots \sum_{l_{j}=0}^{k_{j-1}} \ldots \sum_{l_{r}=0}^{k_{r}}} \\
& \times e_{e_{i j}}\left(l_{1}, l_{2}, \ldots, l_{r}\right) H_{e_{e i j}\left(l_{1}, l_{2}, \ldots, l_{r}\right)}
\end{aligned}
$$

Substitute Eqs. (21) and (22) into Eq. (20), it follows that Eq. (18) is correct.

The proof is completed.

Theorem 3 gives us a computational method about the number of period- $n(n \geq 2)$ orbits. But we are more interested in the number of steady orbits. Theorem 4 gives us the answer.

Theorem 4. Let $I_{N}$ be the number of steady period$n$ ( $n$ is greater than one) orbits, then relationship between $I_{N}$ and $H_{N}$ is

$$
I_{N}=N \cdot \frac{H_{N}}{m+d} .
$$

Proof. The number of steady region can be determined by the center points. So let $g_{N}\left(Z_{i}, C\right)=$ $\prod_{i-1}^{N} f_{Z}\left(Z_{i}, C\right)=0, f_{Z}^{\prime}\left(Z_{i}, C\right)=\left(Z_{i}^{m}+\frac{C}{Z_{i}^{d}}\right)^{\prime}=$
$\frac{1}{Z_{i}^{d+1}}\left(m Z_{i}^{m+d}-c d\right), g_{N}\left(Z_{i}, C\right)=\prod_{i=1}^{N} f_{Z}\left(Z_{i}, C\right)=$ $\prod_{i=1}^{N} \frac{1}{Z_{i}^{d+1}}\left(m Z_{i}^{m+d}-c d\right)$, Then the values of steady center points are $Z_{1}=\left(\frac{d C}{m}\right)^{\frac{1}{m+d}}, Z_{2}=R C^{\frac{m}{m+d}}, Z_{3}=$ $R^{m} C^{\frac{m^{2}}{m+d}}+R^{-d} C^{1-\frac{m d}{m+d}}, \ldots, Z_{N}=R^{m^{N-2}} C^{\frac{m^{N-1}}{m+d}}+$ $\cdots$ and $Z_{N}=R^{m^{N-2}} C^{\frac{m^{N-1}}{m+d}}+\cdots=\left(\frac{d C}{m}\right)^{\frac{1}{m+d}}$, $R=\left(\frac{d}{m}\right)^{\frac{-d}{m+d}}\left(\frac{d}{m}+1\right)$.

Let $C^{\frac{1}{m+d}}=C^{\prime}$, then the formula above can be changed into a $m^{N-1}$ power algebraic equation, which has $m^{N-1}$ roots. Each root has $m+d$ values, so there are $(m+d)^{N-1}$ steady regions. Just like the proof of Theorem 3 we get,

$$
\begin{gathered}
(m+d)^{N-1}=I_{N}+\sum_{i=1}^{r} l_{e i}+\sum_{j=2}^{r} \sum_{i=1}^{j-1} l_{e_{i j}}+\cdots+I_{1} \\
(m+d)^{e_{i}-1}=I_{e_{i}}+\sum_{i=1}^{r} l_{e_{i j}}+\sum_{\substack{k=2 \\
i \neq j \neq k}}^{r} \sum_{j=1}^{k-1} I_{e_{i j k}} \\
\\
+\cdots+I_{1} \\
(m+d)^{e_{i j}-1}= \\
I_{e_{i}}+\sum_{\substack{k=1 \\
i \neq j \neq k}}^{r} I_{e_{i j k}}+\cdots+I_{1} \\
\cdots
\end{gathered}
$$

Then the result is $I_{N}=N \cdot \frac{H_{N}}{m+d}$. This completes the proof of Theorem 4

Theorem 5. The points of McMullen family M sets are conjugate symmetric points.

Proof. We prove the theorem using mathematical induction. Let $c=r e^{i \theta}, \theta \in[0,2 \pi)$, then $\bar{c}=r e^{-i \theta}$. Considering $k=1$,

$$
\begin{aligned}
f^{1}(c) & =r^{m} e^{i m \theta}+r e^{i \theta} r^{-d} e^{-i d \theta} \\
& =r^{m} e^{i m \theta}+r^{1-d} e^{i(1-d) \theta}, \\
f^{1}(\bar{c}) & =r^{m} e^{-i m \theta}+r^{1-d} e^{i(d-1) \theta}, \\
f^{1}(c) & =\overline{f^{1}(\bar{c})} .
\end{aligned}
$$

Suppose $f^{k}(c)=\overline{f^{k}(\bar{c})}$, then

$$
\begin{aligned}
f^{k+1}(C) & =f^{k}\left[f^{1}(C)\right]=\overline{f^{k} \overline{\left[f^{1}(C)\right]}} \\
& =\overline{f^{k}\left[f^{1}(\bar{C})\right]}=\overline{f^{k+1}(\bar{C})} .
\end{aligned}
$$

So using the mathematical induction, Theorem 5 is correct. 


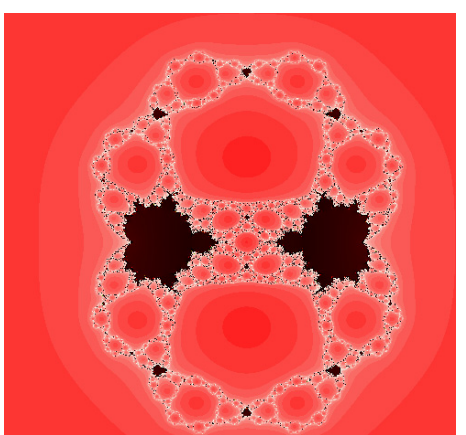

(a) $m=3, d=3$

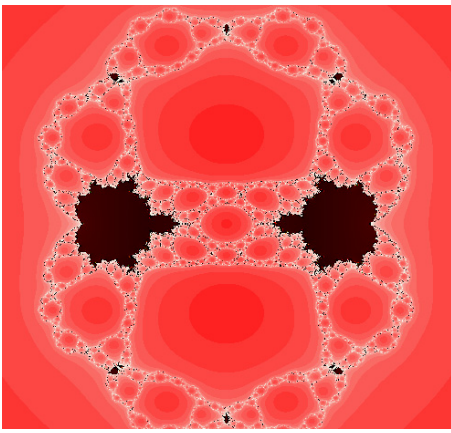

(d) $m=3, d=4$

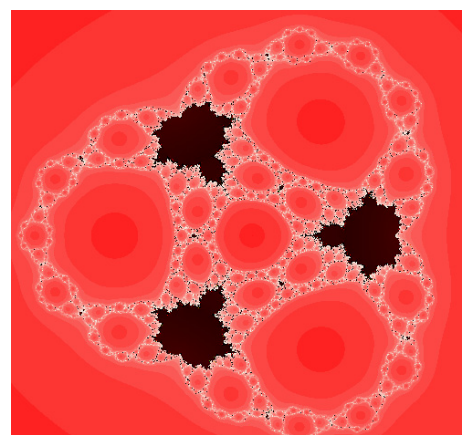

(b) $m=4, d=3$

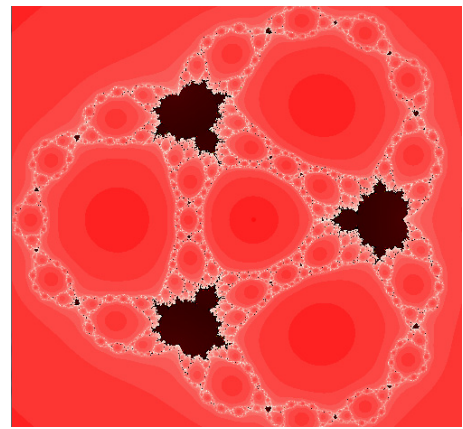

(e) $m=4, d=4$

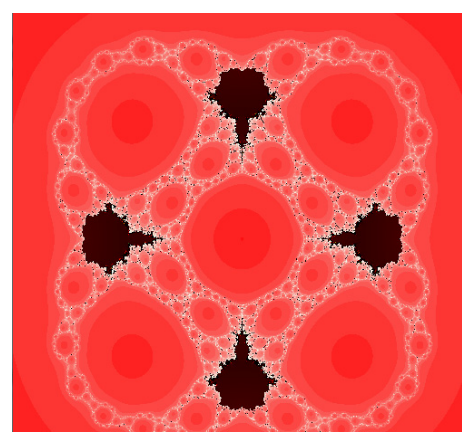

(c) $m=5, d=3$

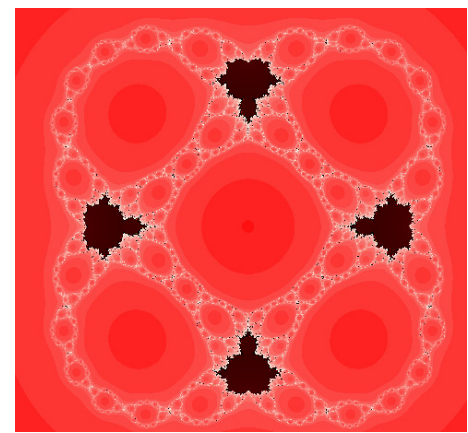

(f) $m=5, d=4$

Fig. $1 n$ family $\mathrm{M}$ sets.

\section{ALGORITHMS DESIGN AND NUMERICAL EXPERIMENTS}

The experiments were conducted on the platform of Microsoft Visual C ++ 6.0. We use escaped time algorithm as the law to judge steady points. The detailed algorithm is given below.

\section{Algorithm 1.}

(1) Initiating the area $W$, choosing $m$ and $d$, which satisfy $\frac{1}{m}+\frac{1}{d}<1,\left(m, d \in N^{+}\right)$.

(2) Let $f_{\lambda}^{\prime}(z)=0$, then we have $m+d$ solutions and choose one of them as a free critical point $z_{0}$.

(3) We choose any point $z$ in the window $W$, beginning with $z_{0}$, iteration under form $f(z)=z^{m}+$ $C / z^{d}$. If $|Z|$ is still less than the escaped radius in the prescriptive time, the point is steady. Otherwise, it is escaped.

(4) We mark steady points as black color and escaped points as red color.

(5) Repeating Steps (3) and (4), until all the points in the area $W$ are covered.

According to Algorithm 1, we have the experiment results as follows.

Figure 1 only shows us part of the experiment results. After plenty of experiments, we find that the number of the largest black regions in every $\mathrm{M}$ set is $m+d-1$. But it does not mean that the number of period- 1 regions is $m+d-1$. We will discuss later that period-1 regions are only a part of them. All the $\mathrm{M}$ sets are conjugate symmetric and each center point is escaped. And for any $M$ set which has the same parameter $m$, the distance among the largest black regions is greater if the value of parameter $d$ is bigger.
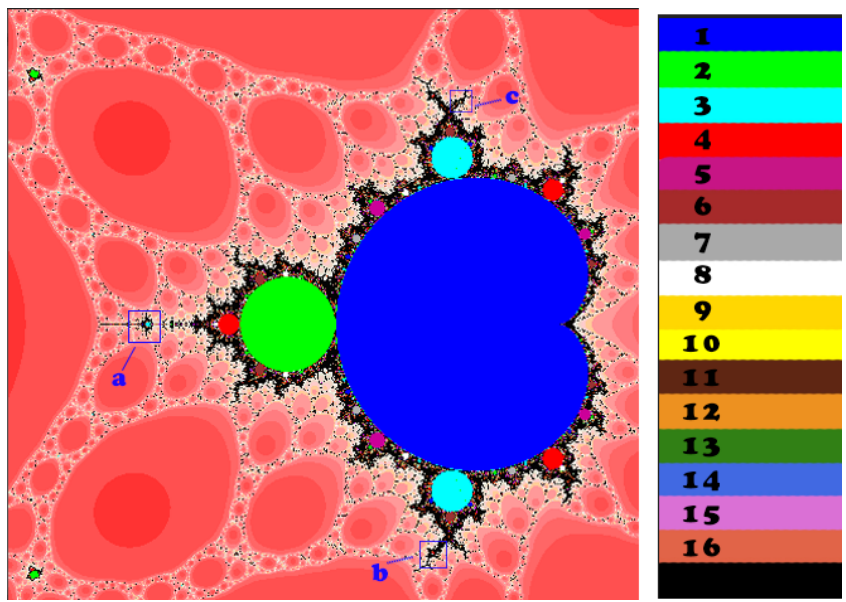

Fig. 2 The color palette. 
Figure 1 shows the whole characteristic of a $\mathrm{M}$ set, but it cannot display the distributions of steady period regions. In this case, we improve Algorithm 1 and change Steps (4) and (5) of Algorithm 1 as follows.

\section{Algorithm 2.}

(4) For each steady point, we continue to iterate it beginning with free critical point $z_{0}$. At the same time, we record the number of the iteration point positions, which is the amount of period.
(5) We mark the steady points with different colors according to the color palette, as shown in Fig. 2

(6) Repeat Steps (4) and (5), until all the points in the area $W$ are covered.

The left part of Fig. 2 shows the amplification of one of the largest steady regions with parameters $m=d=4$. The right part of Fig. 2 is a color palette, according to which we mark period-1 regions with blue and period-2 regions with green.

The experimental results show that different free critical points lead to different results when

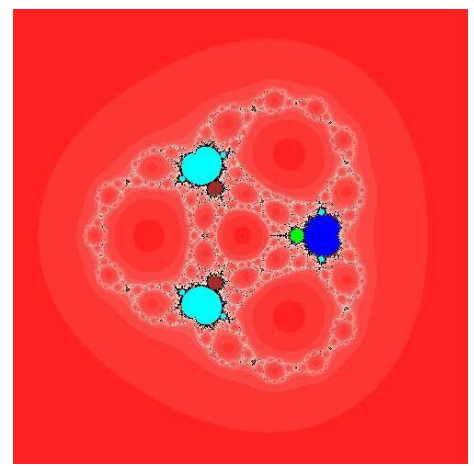

(a) $k=0$

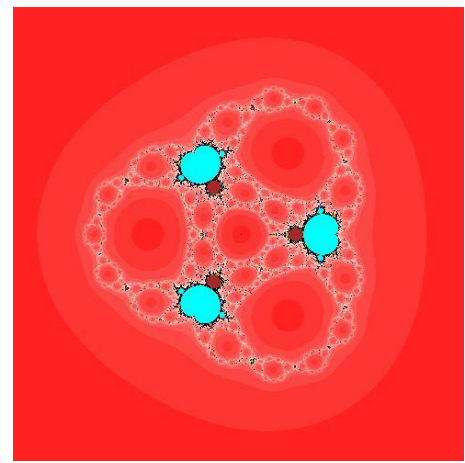

(d) $k=3$

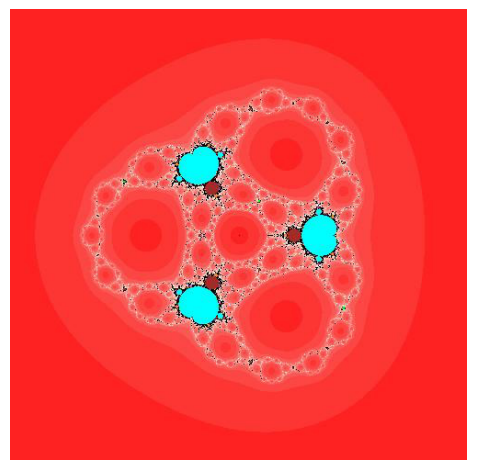

(g) $k=6$

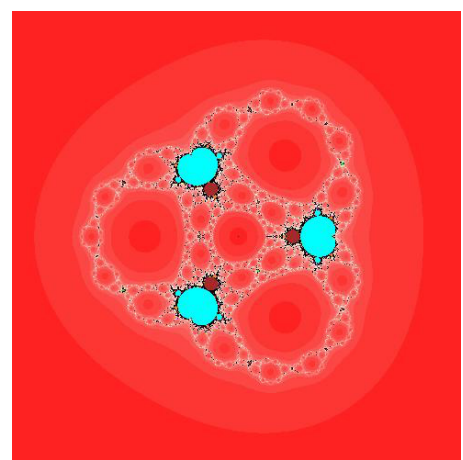

(b) $k=1$

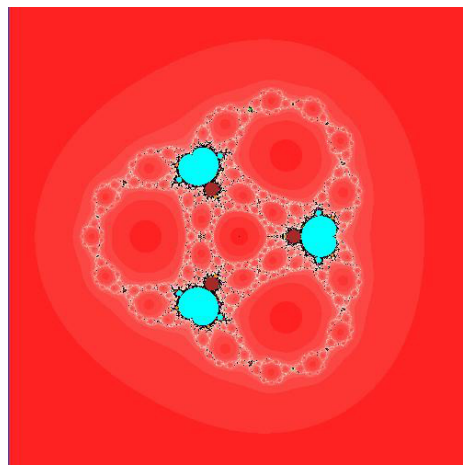

(e) $k=4$

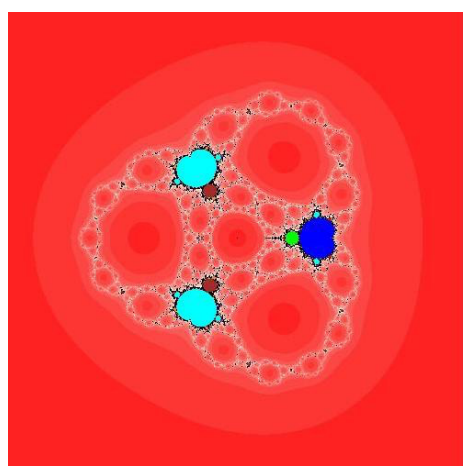

(h) $k=7$

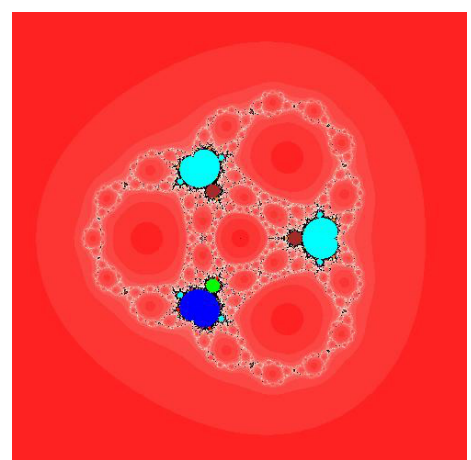

(c) $k=2$

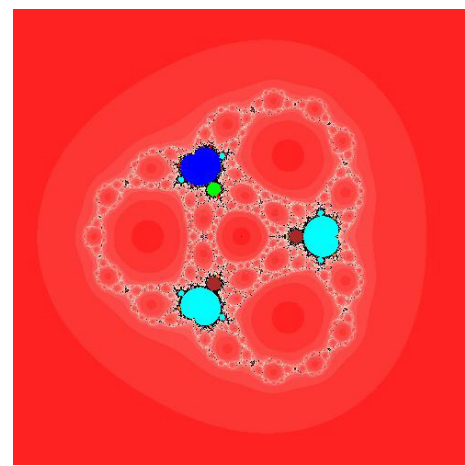

(f) $k=5$

Fig. 3 Different free critical points $\mathrm{M}$ sets $(m=4, d=3)$. 


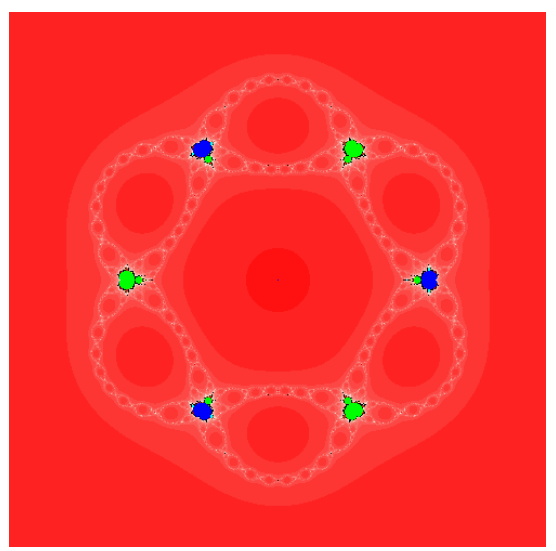

(a) $k=0$

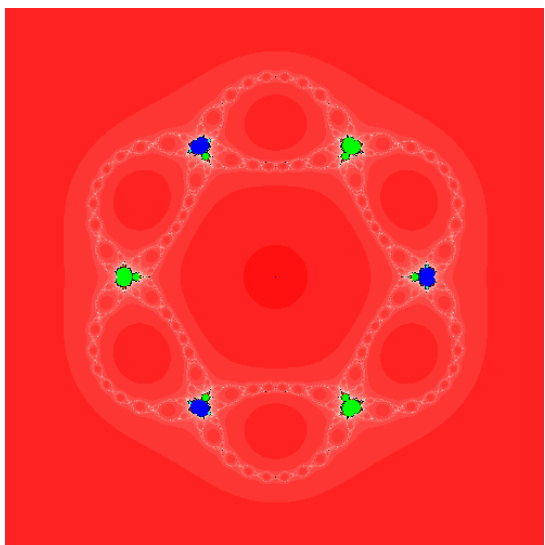

(b) $k=1$

Fig. 4 Different free critical points $\mathrm{M}$ sets $(m=d=7)$.

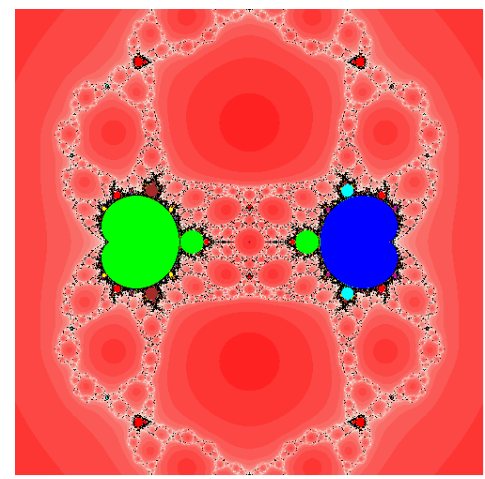

(a) $m=3, d=3$

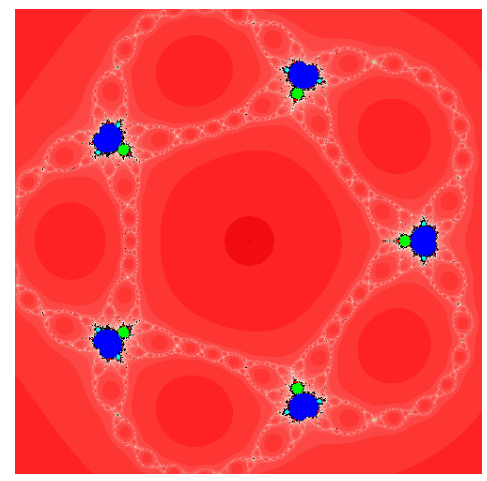

(d) $m=6, d=6$

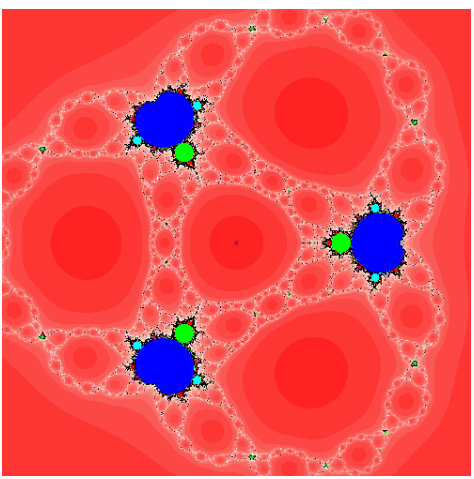

(b) $m=4, d=4$

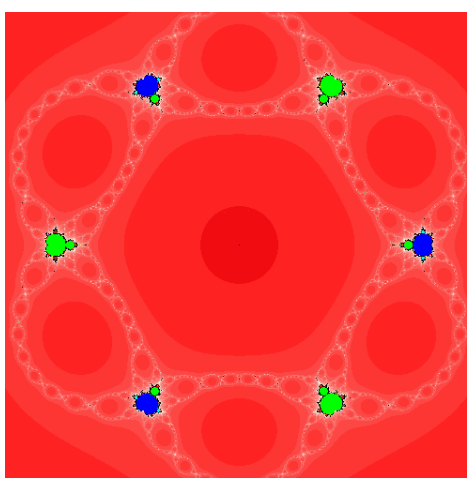

(e) $m=7, d=7$

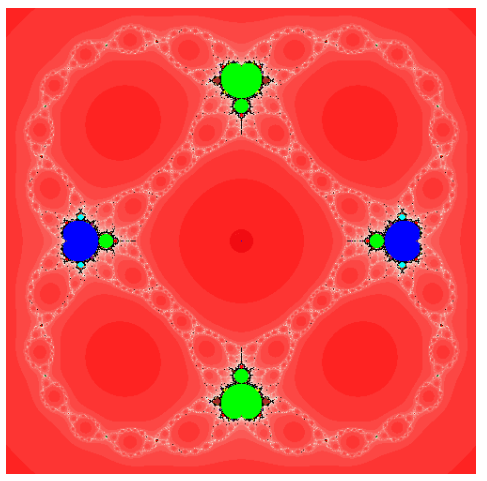

(c) $m=5, d=5$

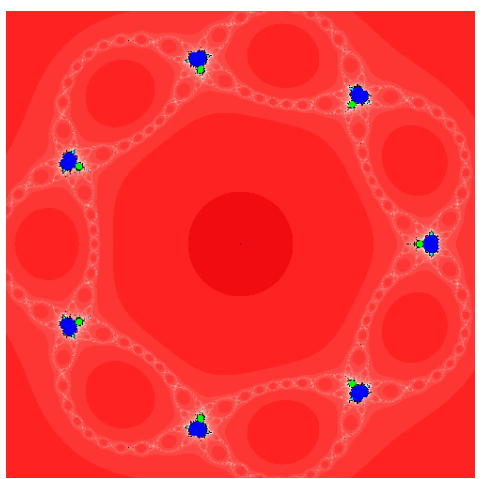

(f) $m=8, d=8$

Fig. 5 McMullen family M sets $(m=d)$.

parameter $m$ and $d$ are not changed, as shown in Fig. 3.

As we know, McMullen family M sets have $m+d$ free critical points. They are $\sqrt[m+d]{\frac{d C}{m}}$. They are infinity or bounded at the same time. When $m=d$, free critical points have no effect on the period regions. A detailed analysis is as follows.

For the form (1), we let $C=r e^{i \theta}, \theta \in[0,2 \pi)$, then the free critical points are $p=(d r / m)^{\frac{1}{m+d}} e^{\frac{\theta+2 k \pi}{m+d}}$, $k \in Z, \theta \in[0,2 \pi)$, where $p$ have $k=0,1, \ldots$, 
$m+d-1, m+d$ points in all. Let the algorithm begin with $Z_{1}=p$, then

$$
\begin{aligned}
& Z_{2}=\left(\frac{d r}{m}\right)^{\frac{m}{m+d}} e^{\frac{(\theta+2 k \pi) m i}{m+d}} \\
&+r e^{i \theta}\left(\frac{d r}{m}\right)^{\frac{-d}{m+d}} e^{\frac{(\theta+2 k \pi)(-d)}{m+d}} \\
&=\left(\frac{d r}{m}\right)^{\frac{m}{m+d}} e^{\frac{i m \theta}{m+d}}\left(1+\frac{m}{d}\right) e^{\frac{-2 k d i \pi}{m+d}}, \\
& k \in Z, \quad \theta \in[0,2 \pi) .
\end{aligned}
$$

It is easy to see that the value of $k$ affects the result. However, when $m=d$. The equation can be changed to $Z_{2}=\left(\frac{r d}{m}\right)^{\frac{m}{m+d}} e^{\frac{m \theta}{m+d}}\left(1+\frac{m}{d}\right) e^{i k \pi}, k \in Z, \theta \in$ $[0,2 \pi)$. So,

$$
Z_{2}=\left\{\begin{array}{cc}
\left(\frac{r d}{m}\right)^{\frac{m}{m+d}} e^{i \frac{m \theta}{m+d}}\left(1+\frac{m}{d}\right), & \\
k=2 n, \quad \theta \in[0,2 \pi), & n \in Z, \\
-\left(\frac{r d}{m}\right)^{\frac{m}{m+d}} e^{i \frac{m \theta}{m+d}}\left(1+\frac{m}{d}\right), & \\
k=2 n+1, \quad \theta \in[0,2 \pi), & n \in Z .
\end{array}\right.
$$

Clearly, the value of $k$ only affects the sign of an iteration result, but not affect the distribution of period regions. It is further verified by the experiment results shown in Fig. 4

Furthermore, when $m=d$, the number of period-1 is

$$
N=\left\{\begin{array}{lll}
m-1, & m=2 n, & n \in Z, \\
\frac{m-1}{2}, & m=2 n+1, & n \in Z .
\end{array}\right.
$$

Proof. We consider the result of Theorem 1

$$
C=m d^{\frac{d+1}{m-1}}(d+m)^{\frac{m+d}{1-m}} e^{\frac{(d+1) 2 k i \pi}{m-1}}, \quad k \in Z .
$$

When $m=d$,

$$
C=m d^{\frac{d+1}{m-1}}(d+m)^{\frac{m+d}{1-m}} e^{\frac{2}{m-1}}, \quad k \in Z .
$$

So, $C$ has $\frac{m-1}{2}$ effective values when $k$ is an odd number, $C$ has $m-1$ values when $k$ is an even number. The experiment results are shown in Fig. 5 .

\section{CONCLUSIONS}

In this study, we use the algebraic analysis method and the stable condition of complex dynamical system to explore the characteristics of McMullen family $\mathrm{M}$ sets. A method of computing the number of period- $n$ steady regions is proposed. The center points and boundaries of period- 1 steady regions are given and the conjugated symmetry structures of the $\mathrm{M}$ set are found.

In addition, we design an algorithm to construct McMullen family $\mathrm{M}$ sets by improving the escaped time algorithm. Different period regions are marked as different colors to distinguish them clearly. According to plenty of experiments, we find that different free critical points lead to distinct results with the same $m$ and $d$. However, the free critical points do not influence the experimental results when $m=d$, which is proved theoretically.

The structures of McMullen family M sets are very complicated, which have prolific and interesting contents. Our study shed light on an example for their studies. There are still lots of appealing and important problems worth further investigations, such as the method of the calculation of center points in period- $n$ steady regions, the influence of free critical points on the structures and period points of the corresponding Julia sets. Future work will focus on the McMullen family Julia sets and high period steady regions of $\mathrm{M}$ sets.

\section{ACKNOWLEDGMENTS}

This research is supported by the National Natural Science Foundation of China (Grant No. 61572103), Scientific Research Fund of Liaoning Provincial Education Department (Grant No. L2014025), and the Fundamental Research Funds for the Central Universities (Grant No. DUT15QY33).

\section{REFERENCES}

1. B. B. Mandelbrot, The Fractal Geometry of Nature (W. H. Freeman and Company, New York, 1983).

2. W. Qiu, X. Wang and Y. Yin, Dynamics of McMullen maps, Adv. Math. 229(4) (2012) $2525-2577$.

3. S. H. Boyd and M. J. Schulz, Geometric limits of Mandelbrot and Julia sets under degree growth, Int. J. Bifurc. Chaos 22(12) (2012) 1250301.

4. H. Qiao, X. Kan and J. Duan, Escape probability for stochastic dynamical systems with jumps, in Malliavin Calculus and Stochastic Analysis (Springer, NY, 2013), 195-216. 
5. Y. Xiao, W. Qiu and Y. Yin, On the dynamics of generalized McMullen maps, Ergod. Theory Dyn. Syst. 34(6) (2014) 2093-2112.

6. X. J. Yang, J. A. T. Machado, C. Cattani and F. Gao, On a fractal LC-electric circuit modeled by local fractional calculus, Commun. Nonlinear Sci. Numer. Simul. 47 (2017) 200-206.

7. X. J. Yang, J. A. T. Machado, D. Baleanu and C. Cattani, On exact traveling-wave solutions for local fractional Korteweg-de Vries equation, Chaos 26(8) (2016) 084312.

8. S. Liu, Z. B. Zhang, L. Y. Qi and M. Ma, A fractal image encoding method based on statistical loss used in agricultural image compression, Multimed. Tools Appl. 75(23) (2016) 15525-15536.

9. S. Liu, X. C. Cheng, W. N. Fu, Y. P. Zhou and Q. Z. Li, Numeric characteristics of generalized M-set with its asymptote, Appl. Math. Comput. 243 (2014) 767-774.

10. S. Liu, W. N. Fu, L. Q. He, J. T. Zhou and M. Ma, Distribution of primary additional errors in fractal encoding method, Multimed. Tools Appl. 76 (2015) $1-16$.

11. S. Liu, X. C. Cheng, C. H. Lan, W. N. Fu, J. T. Zhou, Q. Z. Li and G. L. Gao, Fractal property of generalized M-set with rational number exponent, Appl. Math. Comput. 220 (2013) 668-675.

12. P. Blanchard, R. L. Devaney, D. M. Look, P. Seal and Y. Shapiro, Sierpinski curve Julia sets and singular perturbations of complex polynomials, Ergod. Theory Dyn. Syst. 25(4) (2005) 10471055 .

13. P. Blanchard, R. L. Devaney, D. M. Look, M. Rocha, P. Seal, S. Siegmund and D. Uminsky, Sierpinski carpets and gaskets as Julia sets of rational maps, in Dynamics on the Riemann Sphere (EMS, Denmark, 2006), 97-119.

14. R. L. Devaney, Structure of the McMullen domain in the parameter planes for rational maps, Fundam. Math. 185 (2005) 267-285.

15. R. L. Devaney, Baby Mandelbrot sets adorned with halos in families of rational maps, Contemp. Math. 396 (2006) 37-50.

16. R. L. Devaney, The McMullen domains: Satellite Mandelbrot sets and Sierpinski conform, in Conformal Geometry Dyn. Am. Math. Soc. 11(12) (2007) 164-190.

17. R. L. Devaney and D. M. Look, Symbolic dynamics for Sierpinski curve Julia set, J. Differ. Equ. Appl. 11(7) (2005) 581-596.

18. P. Roesch, On captures for the family $f_{\lambda}(z)=z^{2}+$ $\lambda / z^{2}$, in Dynamics on the Riemann Sphere (EMS, Denmark, 2006), 121-130.

19. Y. Q. Zhong, Theory of Complex Variable Functions (China Higher Education Press, Beijing, 2003).

20. Y. N. Huang and B. S. Xie, An algebraic analysis method looking for the periodic orbit, Adv. Math. 23(2) (1994) 142-148. 